\title{
CRS/AAPS Joint Workshop on Critical Variables in the In Vitro and In Vivo Performance of Parenteral Sustained-Release Products
}

\author{
Michael J. Rathbone', Marilyn N. Martinez², Mai Huynh² ${ }^{2}$, and \\ Diane Burgess ${ }^{3}$ \\ 'School of Pharmacy, Gold Coast Campus, Griffith University, Queensland 4222 Australia \\ 2US Food and Drug Administration, Center for Veterinary Medicine, Rockville, MD 20855 \\ ${ }^{3}$ University of Connecticut, 69 North Eagleville Road, Storrs, CT 06269
}

T he Controlled Release Society (CRS) and the American Association of Pharmaceutical Scientists (AAPS) recently co-sponsored a workshop on the critical variables impacting the in vivo performance of parenteral controlled-release formulations. This workshop was a continuation of discussions initiated during the 2007 CRS Educational Workshop entitled "In Vitro and In Vivo and Considerations Associated with Parenteral Sustained Release Products," the outcome of which was summarized in the Journal of Controlled Release (1). The 2008 workshop focused on the use of design-space concepts as a mechanism to identify the critical quality attributes associated with in vivo product performance and to facilitate the development and use of in vitro test methods for (1) setting batch release characteristics, (2) ensuring the equivalence of product performance when there are changes in formulations, excipients, or manufacturing methods, and (3) modifying batch release specifications. To address these complex issues, the workshop format allowed experts to present their perspectives on challenges associated with assuring the quality and performance of a range of state-of-the art parenteral delivery systems and provided an opportunity to hear and discuss a range of topics critical to parenteral product performance. The ten questions that served as the focus of breakout group discussions are provided in Table 1. It is also noteworthy that this workshop was the first opportunity for CRS membership to consider these questions and provide a response electronically by logging on to the CRS website (2).

Feedback from the workshop participants included the following points:

- Regardless of the type of parenteral product, some methodology should be employed to ensure that it is functioning as intended before batch release.

- In some cases, quality control testing procedures necessitate the use of multiple tests to demonstrate adequate function of these complex delivery systems.

- Host response may not be due solely to the bioactive in the product or to its chemical components, but may

${ }^{1}$ Corresponding author. be related to dosage form size, shape, age, or surface characteristics.

- It is important that product development is not hindered by the process of defining test requirements and that economic and time constraints are considered when developing in vitro release specifications.

- Nontraditional tests can be developed, but relevant release specifications must be set if they are developed. That said, existing USP methods are underutilized, and it would be preferable (prudent) to develop tests that rely on USP apparatus since the specifications for the physical and experimental conditions associated with the use of USP apparatus are well-defined.

- The establishment of any set of product specifications needs to be an iterative process.

- There could be a difference between quality control methods and a method that is used to support a biowaiver. However, if a test is developed solely for quality control purposes and has been designed specifically to monitor critical quality attributes, then it cannot be used to predict biological performance.

- If the release specification is intended to ensure therapeutic activity, it will be necessary to ascertain the biological relevance of the test results and the release specifications through studies that establish the in vivo-in vitro relationship.

- For more complex dosage forms, safety concerns may extend beyond the rate and extent of drug exposure. When there are additional safety or efficacy concerns that cannot be addressed with bioequivalence trials, it may not be feasible to develop generic formulations of these products (e.g., products with safety and activity profiles based on ligand-based targeted delivery systems).

- When employing the design-space concept, extremes of the proposed specifications should be tested both in vitro and in vivo (where in vivo studies can include the use of animal models). The in vivo data are needed to ensure that the in vitro test is reliable and discriminating. When using design space, aged materials need 


\section{Table 1. Workshop Discussion Questions}

1. Is it possible to develop in vitro methods for complex targeted delivery systems?

2. What methods can be used to control host responses to biomaterials?

3. What controls can be used in systems where the host system is an integral component of the targeted drug delivery?

4. Should in vitro release specifications be required for long-acting lipophilic solutions?

5. Should there be a difference between manufacturing and batch release specifications versus those specifications needed to ensure in vivo bioequivalence?

6. What factors should be considered when evaluating whether it is feasible to grant biowaivers for sol-transition modified-release products (i.e., where product is a true solution upon injection)?

7. Despite the possibility of unique formulations that will require variations in standard test methods, is it feasible to develop standardized in vitro test methods that can be applied to any specific type of MR parenteral formulation?

8. When setting expiry for a long-acting parenteral product intended to continue releasing for months upon administration, how does one ensure that the product will perform in a manner comparable to a fresh product if administered at expiry?

9. What unique challenges will be faced when attempting to develop generic versions of parenteral modified-release products? Are there situations when such generic formulations would not be feasible or appropriate?

10. What kinds of in vivo and in vitro data would be needed to support design specifications?

to be included as part of the assessment of critical quality attributes, and the impact of aging on prolonged drug release needs to be assessed.
Because of the great importance of this topic, two future initiatives are underway. A detailed account of the 2008 workshop presentations will be reported in an upcoming theme issue of the AAPS Journal, and a summary report of the breakout discussions will be published in the Journal of Controlled Release. We will inform everyone when these publications are available.

The consensus from workshop participants was that although there is great need to develop and validate meaningful release specifications, it is important to recognize the distinction between clinically important information (to ensure product quality) and performance and the imposition of study requirements that would serve primarily as a focus of scientific inquiry. While the former is critical for ensuring public health and confidence, the latter could impede progress toward the development of novel delivery technologies and dosage forms that meet medical needs.

\section{REFERENCES}

1. Martinez, M.; Rathbone, M.; Burgess, D.; Huynh, M. In vitro and in vivo considerations associated with parenteral sustained release products: A review based upon information presented and points expressed at the 2007 Controlled Release Society Annual Meeting. J. Controlled Release 2008, 129 (2), 79-87.

2. Martinez, M.; Huynh, M.; Burgess, D.; Rathbone, M. Defining the Critical Quality Attributes of Modified Release Parenteral Dosage Forms: Opportunities for Ongoing Dialogue. CRS Newsletter 2008, 25 (3), 20-21. 